\title{
Hermite-Hadamard type inequalities for product of GA-convex functions via Hadamard fractional integrals
}

\author{
İmdat İşcan and Mehmet Kunt
}

\begin{abstract}
In this paper, some Hermite-Hadamard type inequalities for products of two GA-convex functions via Hadamard fractional integrals are established. Our results about GA-convex functions are analogous generalizations for some other results proved by Pachpette for convex functions.
\end{abstract}

Mathematics Subject Classification (2010): 26A51, 26A33, 26D10.

Keywords: Hermite-Hadamard inequality, GA-convex functions, Hadamard fractional integral.

\section{Introduction}

In recent years, very large number of studies of error estimations have been done for Hermite-Hadamard type inequalities. It is known that Hermite-hadamard integral inequality was built on a convex function. In time, Hermite-Hadamrd inequality is developed other kinds of convex functions. For some results which generalize, improve, and extend the Hermite-Hadamard inequality see $[1,7,10,18,20]$ and references therein.

Hermite-Hadamard type inequalities for products of two convex functions are interesting problem and firstly developed by Pachpatte in [16]. In [17], Pachpette also established Hermite-hadamard type inequalities involving two log-convex functions. In [11], Kırmacı et. al. proved several Hermite-Hadamard type inequalities for products of two convex and $s$-convex functions. In [19], Sarıkaya et. al. proved some HermiteHadamard type inequalities for products of two $h$-convex functions. In [2], Bakula et. al. established Hermite-Hadamard type inequalities for products of two $m$-convex and $(\alpha, m)$-convex functions. In $[4,6]$, Chen and $\mathrm{Wu}$ obtained some Hermite-Hadamard type inequalities for products of two convex and harmonically $s$-convex functions. In [21], Yin and Qi established some Hermite-Hadamard type inequalities for products 
of two convex functions. In [5], Chen obtained some new Hermite-Hadamard type inequalities for products of two convex functions via Riemann-Liouville fractional integrals and in [3] he extended this problem to $m$-convex and $(\alpha, m)$-convex functions.

In this work, we establish Hermite-Hadamard type inequalities for products of two GA-convex functions via Hadamard fractional integrals. Our results are analogous generalization for some results in [16].

\section{Preliminaries}

Let $f: I \subseteq \mathbb{R} \rightarrow \mathbb{R}$ be a convex function defined on the interval $I$ of real numbers and $a, b \in I$ with $a<b$. The inequality

$$
f\left(\frac{a+b}{2}\right) \leq \frac{1}{b-a} \int_{a}^{b} f(x) d x \leq \frac{f(a)+f(b)}{2}
$$

is well known in the literature as Hermite-Hadamard's inequality [8].

In [16], Pachpette established following two Hermite-Hadamard type inequalities for products of convex functions as follows:

Theorem 2.1. Let $f$ and $g$ be real-valued, non-negative and convex functions on $[a, b]$. Then

$$
\frac{1}{b-a} \int_{a}^{b} f(x) g(x) d x \leq \frac{1}{3} M(a, b)+\frac{1}{6} N(a, b)
$$

and

$$
\begin{aligned}
2 f\left(\frac{a+b}{2}\right) g\left(\frac{a+b}{2}\right) \leq & \frac{1}{b-a} \int_{a}^{b} f(x) g(x) d x \\
& +\frac{1}{6} M(a, b)+\frac{1}{3} N(a, b)
\end{aligned}
$$

where $M(a, b)=f(a) g(a)+f(b) g(b)$ and $N(a, b)=f(a) g(b)+f(b) g(a)$.

Definition 2.2. $[14,15]$. A function $f: I \subseteq(0, \infty) \rightarrow \mathbb{R}$ is said to be GA-convex (geometric-arithmetically convex) if

$$
f\left(x^{t} y^{1-t}\right) \leq t f(x)+(1-t) f(y)
$$

for all $x, y \in I$ and $t \in[0,1]$.

We will now give definitions of the right-hand side and left-hand side Hadamard fractional integrals which are used throughout this paper.

Definition 2.3. [12]. Let $f \in L[a, b]$. The right-hand side and left-hand side Hadamard fractional integrals $J_{a+}^{\alpha} f$ and $J_{b-}^{\alpha} f$ of order $\alpha>0$ with $b>a \geq 0$ are defined by

$$
J_{a+}^{\alpha} f(x)=\frac{1}{\Gamma(\alpha)} \int_{a}^{x}\left(\ln \frac{x}{t}\right)^{\alpha-1} f(t) \frac{d t}{t}, x>a
$$

and

$$
J_{b-}^{\alpha} f(x)=\frac{1}{\Gamma(\alpha)} \int_{x}^{b}\left(\ln \frac{t}{x}\right)^{\alpha-1} f(t) \frac{d t}{t}, x<b
$$


respectively, where $\Gamma(\alpha)$ is the Gamma function defined by

$$
\Gamma(\alpha)=\int_{0}^{\infty} e^{-t} t^{\alpha-1} d t
$$

In [9], İşcan represented Hermite-Hadamard's inequalities for GA-convex functions in fractional integral forms as follows.

Theorem 2.4. Let $f: I \subseteq(0, \infty) \rightarrow \mathbb{R}$ be a function such that $f \in L[a, b]$ where $a, b \in I$ with $a<b$. If $f$ is a GA-convex function on $[a, b]$, then the following inequalities for fractional integrals hold:

$$
f(\sqrt{a b}) \leq \frac{\Gamma(\alpha+1)}{2\left(\ln \frac{b}{a}\right)^{\alpha}}\left[J_{a+}^{\alpha} f(b)+J_{b-}^{\alpha} f(a)\right] \leq \frac{f(a)+f(b)}{2}
$$

with $\alpha>0$.

In [13], Kunt and İşcan established new Hermite-Hadamard type inequality for GA-convex function in fractional integral forms as follows:

Theorem 2.5. Let $f:[a, b] \subseteq(0, \infty) \rightarrow \mathbb{R}$ be a GA-convex function with $a<b$ and $f \in L[a, b]$, then the following inequalities for fractional integrals hold:

$$
f(\sqrt{a b}) \leq \frac{\Gamma(\alpha+1)}{2^{1-\alpha}\left(\ln \frac{b}{a}\right)^{\alpha}}\left[J_{\sqrt{a b}-}^{\alpha} f(a)+J_{\sqrt{a b}+}^{\alpha} f(b)\right] \leq \frac{f(a)+f(b)}{2} .
$$

\section{General results}

Theorem 3.1. Let $f$ and $g:[a, b] \subseteq(0, \infty) \rightarrow \mathbb{R}$ be non-negative and GA-convex functions with $a<b$ and $f \in L[a, b]$, then the following inequality for fractional integrals hold:

$$
\begin{aligned}
& \frac{\Gamma(\alpha+1)}{2\left(\ln \frac{b}{a}\right)^{\alpha}}\left[J_{a+}^{\alpha} f(b) g(b)+J_{b-}^{\alpha} f(a) g(a)\right] \\
\leq & \left(\frac{\alpha}{\alpha+2}-\frac{\alpha}{\alpha+1}+\frac{1}{2}\right) M(a, b)+\frac{\alpha}{(\alpha+2)(\alpha+1)} N(a, b)
\end{aligned}
$$

where $\alpha>0, M(a, b)=f(a) g(a)+f(b) g(b)$ and $N(a, b)=f(a) g(b)+f(b) g(a)$.

Proof. Since $f$ and $g$ are non-negative and GA-convex functions on $[a, b]$, we have for all $t \in[0,1]$

$$
f\left(a^{t} b^{1-t}\right) \leq t f(a)+(1-t) f(b)
$$

and

$$
g\left(a^{t} b^{1-t}\right) \leq t g(a)+(1-t) g(b) .
$$

From products of (3.2) and (3.3), we have

$$
\begin{aligned}
f\left(a^{t} b^{1-t}\right) g\left(a^{t} b^{1-t}\right) \leq & t^{2} f(a) g(a)+(1-t)^{2} f(b) g(b) \\
& +t(1-t)[f(a) g(b)+f(b) g(a)] .
\end{aligned}
$$


Similarly (3.4), we have

$$
\begin{aligned}
f\left(a^{1-t} b^{t}\right) g\left(a^{1-t} b^{t}\right) \leq & (1-t)^{2} f(a) g(a)+t^{2} f(b) g(b) \\
& +t(1-t)[f(a) g(b)+f(b) g(a)] .
\end{aligned}
$$

The sum of (3.4) and (3.5), we have

$$
\begin{aligned}
& f\left(a^{t} b^{1-t}\right) g\left(a^{t} b^{1-t}\right)+f\left(a^{1-t} b^{t}\right) g\left(a^{1-t} b^{t}\right) \\
\leq & \left(2 t^{2}-2 t+1\right) M(a, b)+2 t(1-t) N(a, b)
\end{aligned}
$$

Multiplying both sides of (3.6) by $t^{\alpha-1} \frac{\alpha}{2}$, then integrating the obtained inequality with respect to $t$ over $[0,1]$, we have

$$
\begin{aligned}
& \frac{\alpha}{2}\left[\int_{0}^{1} t^{\alpha-1} f\left(a^{t} b^{1-t}\right) g\left(a^{t} b^{1-t}\right) d t+\int_{0}^{1} t^{\alpha-1} f\left(a^{1-t} b^{t}\right) g\left(a^{1-t} b^{t}\right) d t\right] \\
= & \frac{\alpha}{2}\left[\int_{a}^{b}\left(\frac{\ln \frac{b}{u}}{\ln \frac{b}{a}}\right)^{\alpha-1} f(u) g(u) \frac{d u}{u \ln \frac{b}{a}}+\int_{a}^{b}\left(\frac{\ln \frac{v}{a}}{\ln \frac{b}{a}}\right)^{\alpha-1} f(v) g(v) \frac{d u}{v \ln \frac{b}{a}}\right] \\
= & \frac{\alpha}{2\left(\ln \frac{b}{a}\right)^{\alpha}}\left[\int_{a}^{b}\left(\ln \frac{b}{u}\right)^{\alpha-1} f(u) g(u) \frac{d u}{u}+\int_{a}^{b}\left(\ln \frac{v}{a}\right)^{\alpha-1} f(v) g(v) \frac{d u}{v}\right] \\
= & \frac{\Gamma(\alpha+1)}{2\left(\ln \frac{b}{a}\right)^{\alpha}}\left[J_{a+}^{\alpha} f(b) g(b)+J_{b-}^{\alpha} f(a) g(a)\right] \\
\leq & \frac{\alpha}{2}\left[M(a, b) \int_{0}^{1} t^{\alpha-1}\left(2 t^{2}-2 t+1\right) d t+N(a, b) \int_{0}^{1} t^{\alpha-1} 2 t(1-t) d t\right] \\
= & \left(\frac{\alpha}{\alpha+2}-\frac{\alpha}{\alpha+1}+\frac{1}{2}\right) M(a, b)+\frac{\alpha}{(\alpha+2)(\alpha+1)} N(a, b)
\end{aligned}
$$

and this completes the proof.

Remark 3.2. Theorem 3.1 is an analogous generalization of (2.2) for GA-convex functions.

Corollary 3.3. In Theorem 3.1 , if we take $g:[a, b] \rightarrow \mathbb{R}$ as $g(x)=1$ for all $x \in[a, b]$, then we have

$$
\frac{\Gamma(\alpha+1)}{2\left(\ln \frac{b}{a}\right)^{\alpha}}\left[J_{a+}^{\alpha} f(b)+J_{b-}^{\alpha} f(a)\right] \leq \frac{f(a)+f(b)}{2}
$$

which is the right hand side of (2.4).

Corollary 3.4. In Theorem 3.1, if we take $\alpha=1$, then we have

$$
\frac{1}{\ln b-\ln a} \int_{a}^{b} f(x) g(x) \frac{d x}{x} \leq \frac{1}{3} M(a, b)+\frac{1}{6} N(a, b)
$$

for GA-convex functions. 
Theorem 3.5. Let $f$ and $g:[a, b] \subseteq(0, \infty) \rightarrow \mathbb{R}$ be non-negative and GA-convex functions with $a<b$ and $f \in L[a, b]$, then the following inequality for fractional integrals hold:

$$
\begin{aligned}
& 2 f(\sqrt{a b}) g(\sqrt{a b}) \leq \frac{\Gamma(\alpha+1)}{2\left(\ln \frac{b}{a}\right)^{\alpha}}\left[J_{a+}^{\alpha} f(b) g(b)+J_{b-}^{\alpha} f(a) g(a)\right] \\
& +\frac{\alpha}{(\alpha+2)(\alpha+1)} M(a, b)+\left(\frac{\alpha}{\alpha+2}-\frac{\alpha}{\alpha+1}+\frac{1}{2}\right) N(a, b)
\end{aligned}
$$

where $\alpha>0, M(a, b)=f(a) g(a)+f(b) g(b)$ and $N(a, b)=f(a) g(b)+f(b) g(a)$. Proof. It is clear for all $t \in[0,1]$

$$
\sqrt{a b}=\sqrt{a^{t} b^{1-t} \cdot a^{1-t} b^{t}}=\sqrt{a^{t} b^{1-t}} \sqrt{a^{1-t} b^{t}} .
$$

Since $f$ and $g$ are non-negative and GA-convex functions on $[a, b]$, we have for all $t \in[0,1]$

$$
\begin{aligned}
f(\sqrt{a b}) g(\sqrt{a b})= & f\left(\sqrt{a^{t} b^{1-t}} \sqrt{a^{1-t} b^{t}}\right) g\left(\sqrt{a^{t} b^{1-t}} \sqrt{a^{1-t} b^{t}}\right) \\
\leq & \frac{1}{4}\left[f\left(a^{t} b^{1-t}\right)+f\left(a^{1-t} b^{t}\right)\right]\left[g\left(a^{t} b^{1-t}\right)+g\left(a^{1-t} b^{t}\right)\right] \\
= & \frac{1}{4}\left[f\left(a^{t} b^{1-t}\right) g\left(a^{t} b^{1-t}\right)+f\left(a^{1-t} b^{t}\right) g\left(a^{1-t} b^{t}\right)\right] \\
& +\frac{1}{4}\left[f\left(a^{t} b^{1-t}\right) g\left(a^{1-t} b^{t}\right)+f\left(a^{1-t} b^{t}\right) g\left(a^{t} b^{1-t}\right)\right] \\
\leq & \frac{1}{4}\left[f\left(a^{t} b^{1-t}\right) g\left(a^{t} b^{1-t}\right)+f\left(a^{1-t} b^{t}\right) g\left(a^{1-t} b^{t}\right)\right] \\
& +\frac{1}{4}[t f(a)+(1-t) f(b)][(1-t) g(a)+t g(b)] \\
& +\frac{1}{4}[(1-t) f(a)+t f(b)][t g(a)+(1-t) g(b)] \\
= & \frac{1}{4}\left[f\left(a^{t} b^{1-t}\right) g\left(a^{t} b^{1-t}\right)+f\left(a^{1-t} b^{t}\right) g\left(a^{1-t} b^{t}\right)\right] \\
& +\frac{1}{4}\{2 t(1-t)[f(a) g(a)+f(b) g(b)] \\
& \left.+\left(2 t^{2}-2 t+1\right)[f(a) g(b)+f(b) g(a)]\right\}
\end{aligned}
$$

Multiplying both sides of (3.8) by $2 \alpha t^{\alpha-1}$, then integrating the obtained inequality with respect to $t$ over $[0,1]$, we have

$$
\begin{aligned}
2 f(\sqrt{a b}) g(\sqrt{a b}) \leq & \frac{\Gamma(\alpha+1)}{2\left(\ln \frac{b}{a}\right)^{\alpha}}\left[J_{a+}^{\alpha} f(b) g(b)+J_{b-}^{\alpha} f(a) g(a)\right] \\
& +\frac{\alpha}{(\alpha+2)(\alpha+1)} M(a, b)+\left(\frac{\alpha}{\alpha+2}-\frac{\alpha}{\alpha+1}+\frac{1}{2}\right) N(a, b)
\end{aligned}
$$

and this completes the proof.

Remark 3.6. Theorem 3.5 is an analogous generalization of (2.3) for GA-convex functions. 
Corollary 3.7. In Theorem 3.5 , if we take $g:[a, b] \rightarrow \mathbb{R}$ as $g(x)=1$ for all $x \in[a, b]$, then we have

$$
2 f(\sqrt{a b}) \leq \frac{\Gamma(\alpha+1)}{2\left(\ln \frac{b}{a}\right)^{\alpha}}\left[J_{a+}^{\alpha} f(b)+J_{b-}^{\alpha} f(a)\right]+\frac{f(a)+f(b)}{2} .
$$

Corollary 3.8. In Theorem 3.5, if we take $\alpha=1$, then we have

$$
2 f(\sqrt{a b}) g(\sqrt{a b}) \leq \frac{1}{\ln b-\ln a} \int_{a}^{b} f(x) g(x) \frac{d x}{x}+\frac{1}{6} M(a, b)+\frac{1}{3} N(a, b)
$$

for GA-convex functions.

Theorem 3.9. Let $f$ and $g:[a, b] \subseteq(0, \infty) \rightarrow \mathbb{R}$ be non-negative and GA-convex functions with $a<b$ and $f \in L[a, b]$, then the following inequality for fractional integrals hold:

$$
\begin{aligned}
& \frac{\Gamma(\alpha+1)}{2^{1-\alpha}\left(\ln \frac{b}{a}\right)^{\alpha}}\left[J_{\sqrt{a b}-}^{\alpha} f(a) g(a)+J_{\sqrt{a b}+}^{\alpha} f(b) g(b)\right] \\
\leq & \left(\frac{\alpha}{4(\alpha+2)}-\frac{\alpha}{2(\alpha+1)}+\frac{1}{2}\right) M(a, b)+\frac{\alpha^{2}+3 \alpha}{4(\alpha+2)(\alpha+1)} N(a, b)
\end{aligned}
$$

where $\alpha>0, M(a, b)=f(a) g(a)+f(b) g(b)$ and $N(a, b)=f(a) g(b)+f(b) g(a)$. Proof. Since $f$ and $g$ are non-negative and GA-convex functions on $[a, b]$, multiplying both sides of (3.6) by $t^{\alpha-1} \frac{\alpha}{2^{1-\alpha}}$, then integrating the obtained inequality with respect to $t$ over $\left[0, \frac{1}{2}\right]$, we have

$$
\begin{aligned}
& \frac{\alpha}{2^{1-\alpha}}\left[\int_{0}^{\frac{1}{2}} t^{\alpha-1} f\left(a^{t} b^{1-t}\right) g\left(a^{t} b^{1-t}\right) d t+\int_{0}^{\frac{1}{2}} t^{\alpha-1} f\left(a^{1-t} b^{t}\right) g\left(a^{1-t} b^{t}\right) d t\right] \\
= & \frac{\alpha}{2^{1-\alpha}}\left[\int_{\sqrt{a b}}^{b}\left(\frac{\ln \frac{b}{u}}{\ln \frac{b}{a}}\right)^{\alpha-1} f(u) g(u) \frac{d u}{u \ln \frac{b}{a}}+\int_{a}^{\sqrt{a b}}\left(\frac{\ln \frac{v}{a}}{\ln \frac{b}{a}}\right)^{\alpha-1} f(v) g(v) \frac{d u}{v \ln \frac{b}{a}}\right] \\
= & \frac{\alpha}{2^{1-\alpha}\left(\ln \frac{b}{a}\right)^{\alpha}}\left[\int_{\sqrt{a b}}^{b}\left(\ln \frac{b}{u}\right)^{\alpha-1} f(u) g(u) \frac{d u}{u}+\int_{a}^{\sqrt{a b}}\left(\ln \frac{v}{a}\right)^{\alpha-1} f(v) g(v) \frac{d u}{v}\right] \\
= & \frac{\Gamma(\alpha+1)}{2^{1-\alpha}\left(\ln \frac{b}{a}\right)^{\alpha}}\left[J_{\sqrt{a b}+}^{\alpha}(b) g(b)+J_{\sqrt{a b}-}^{\alpha} f(a) g(a)\right] \\
\leq & \frac{\alpha}{2^{1-\alpha}}\left[M(a, b) \int_{0}^{\frac{1}{2}} t^{\alpha-1}\left(2 t^{2}-2 t+1\right) d t+N(a, b) \int_{0}^{\frac{1}{2}} t^{\alpha-1} 2 t(1-t) d t\right] \\
= & \left(\frac{\alpha}{4(\alpha+2)}-\frac{\alpha}{2(\alpha+1)}+\frac{1}{2}\right) M(a, b)+\frac{\alpha^{2}+3 \alpha}{4(\alpha+2)(\alpha+1)} N(a, b)
\end{aligned}
$$

and this completes the proof.

Remark 3.10. Theorem 3.9 is an other analogous generalization of (2.2) for GA-convex functions. 
Corollary 3.11. In Theorem 3.9, if we take $g:[a, b] \rightarrow \mathbb{R}$ as $g(x)=1$ for all $x \in[a, b]$, then we have

$$
\frac{\Gamma(\alpha+1)}{2^{1-\alpha}\left(\ln \frac{b}{a}\right)^{\alpha}}\left[J_{\sqrt{a b}-}^{\alpha} f(a)+J_{\sqrt{a b}+}^{\alpha} f(b)\right] \leq \frac{f(a)+f(b)}{2}
$$

which is the right hand side of (2.5).

Corollary 3.12. In Theorem 3.9, if we take $\alpha=1$, then we have

$$
\frac{1}{\ln b-\ln a} \int_{a}^{b} f(x) g(x) \frac{d x}{x} \leq \frac{1}{3} M(a, b)+\frac{1}{6} N(a, b)
$$

for GA-convex functions.

Theorem 3.13. Let $f$ and $g:[a, b] \subseteq(0, \infty) \rightarrow \mathbb{R}$ be non-negative and GA-convex functions with $a<b$ and $f \in L[a, b]$, then the following inequality for fractional integrals hold:

$$
\begin{aligned}
& 2 f(\sqrt{a b}) g(\sqrt{a b}) \leq \frac{\Gamma(\alpha+1)}{2^{1-\alpha}\left(\ln \frac{b}{a}\right)^{\alpha}}\left[J_{\sqrt{a b}-}^{\alpha} f(a) g(a)+J_{\sqrt{a b}+}^{\alpha} f(b) g(b)\right] \\
& +\frac{\alpha^{2}+3 \alpha}{4(\alpha+2)(\alpha+1)} M(a, b)+\left(\frac{\alpha}{4(\alpha+2)}-\frac{\alpha}{2(\alpha+1)}+\frac{1}{2}\right) N(a, b)
\end{aligned}
$$

where $\alpha>0, M(a, b)=f(a) g(a)+f(b) g(b)$ and $N(a, b)=f(a) g(b)+f(b) g(a)$. Proof. Multiplying both sides of (3.8) by $2^{1+\alpha} \alpha t^{\alpha-1}$, then integrating the obtained inequality with respect to $t$ over $\left[0, \frac{1}{2}\right]$, we have desired result.

Remark 3.14. Theorem 3.13 is an other analogous generalization of (2.3) for GAconvex functions.

Corollary 3.15. In Theorem 3.13, if we take $g:[a, b] \rightarrow \mathbb{R}$ as $g(x)=1$ for all $x \in[a, b]$, then we have

$$
2 f(\sqrt{a b}) \leq \frac{\Gamma(\alpha+1)}{2^{1-\alpha}\left(\ln \frac{b}{a}\right)^{\alpha}}\left[J_{\sqrt{a b}-}^{\alpha} f(a)+J_{\sqrt{a b}+}^{\alpha} f(b)\right]+\frac{f(a)+f(b)}{2} .
$$

Corollary 3.16. In Theorem 3.13, if we take $\alpha=1$, then we have

$$
2 f(\sqrt{a b}) g(\sqrt{a b}) \leq \frac{1}{\ln b-\ln a} \int_{a}^{b} f(x) g(x) \frac{d x}{x}+\frac{1}{6} M(a, b)+\frac{1}{3} N(a, b)
$$

for GA-convex functions.

\section{References}

[1] Bai, S.P., Wang, S.H., Qi, F., Some Hermite-Hadamard type inequalities for $n$-time differentiable $(\alpha, m)$-convex functions, J. Inequal. Appl., 267(2012), 11 pages.

[2] Bakula, M.K., Özdemir, M.E., Pečari'c, J., Hadamard type inequalities form m-convex and $(\alpha, m)$-convex functions, Journal of Inequalities in Pure and Applied Mathematics, $\mathbf{9}(2008)$, no. 4 , article 96 . 
[3] Chen, F., On Hermite-Hadamard type inequalities for Riemann-Liouville fractional integrals via two kinds of convexity, Chinese Journal of Mathematics, Volume 2014, Article ID:173293, 2014.

[4] Chen, F., A note on Hermite-Hadamard inequalities for products of convex functions, Journal of Applied Mathematics, Volume 2013, Article ID:935020, 2013.

[5] Chen, F., A note on Hermite-Hadamard inequalities for products of convex functions via Riemann-Liouville fractional integrals, Italian Journal of Pure and Applied Mathematics, 33(2014), 299-306.

[6] Chen, F., Wu, S., Some Hermite-Hadamard type inequalities for harmonically s-convex functions, The Scientific World Journal, Vol. 2014, Art. ID:279158, 2014.

[7] Dragomir, S.S., Refinements of the Hermite-Hadamard integral inequality for log-convex functions, Aust. Math. Soc. Gaz., 28(2001), no. 3, 129-134.

[8] Hadamard, J., Étude sur les propriétés des fonctions entières et en particulier d'une fonction considérée par Riemann, J. Math. Pures Appl., 58(1983), 171-215.

[9] İşcan, İ., New general integral inequalities for quasi-geometrically convex functions via fractional integrals, J. Inequal. Appl., 491(2013), 15 pages.

[10] İşcan, İ., Hermite-Hadamard type inequalities for harmonically convex functions, Hacet. J. Math. Stat., 43(2014), no. 6, 935-942.

[11] Kırmacı, U.S., Bakula, M.K., Özdemir, M.E., Pečari'c, J., Hadamard-type inequalities for s-convex functions, Applied Mathematics and Computation, 193(2007), no. 1, 26-35.

[12] Kilbas, A.A., Srivastava, H.M., Trujillo, J.J., Theory and applications of fractional differential equations, Elsevier, Amsterdam, 2006.

[13] Kunt, M., İşcan, İ., On new inequalities of Hermite-Hadamard-Fejer type for GA-convex functions via fractional integrals, RGMIA Research Report Collection, 18(2015), Art. 108, 12 pp.

[14] Niculescu, C.P., Convexity according to the geometric mean, Math. Inequal. Appl., 3(2000), no. 2, 155-167.

[15] Niculescu, C.P., Convexity according to means, Math. Inequal. Appl., 6(2003), no. 4, 571-579.

[16] Pachpatte, B.G., On some inequalities for convex functions, RGMIA Research Report Collection E, vol. 6, 2003.

[17] Pachpatte, B.G., A note on integral inequalities involving two log-convex functions, Mathematical Inequalities and Applications, 7(2004), no. 4, 511-515.

[18] Rubinov, A.M., Dutta, J., Hadamard inequality for quasi-convex functions in higher dimensions, J. Math. Anal. Appl., 270(2002), 80-91.

[19] Sarıkaya, M.Z., Sağlam, A., Yıldırım, H., On some Hadamard-type inequalities for $h$ convex functions, Journal of Mathematical Inequalities, 2(2008), no. 3, 335-341.

[20] Yang, G.S., Refinements of Hadamard inequality for $r$-convex functions, Indian J. Pure Appl. Math., 32(2001), no. 10, 1571-1579.

[21] Yin, H.P., Qi, F., Hermite-Hadamard type inequalities for the product of $(\alpha, m)$-convex functions, Journal of Nonlinear Science and Applications, 8(2015), 231-236. 
Imdat Işsan

Department of Mathematics

Faculty of Sciences and Arts

Giresun University

Giresun, Turkey

e-mail: imdat.iscan@giresun.edu.tr; imdati@yahoo.com

Mehmet Kunt

Department of Mathematics

Faculty of Sciences

Karadeniz Technical University

Trabzon, Turkey

e-mail: mkunt@ktu.edu.tr 